\title{
Automatic License Plate Localization Using Intrinsic Rules Saliency
}

\author{
Chirag N. Paunwala \\ Asst. Prof, Electronics and Communication Engg Dept \\ Sarvajanik College of Engg and Tech. \\ Surat, India
}

\author{
Dr. Suprava Patnaik \\ Professor, Electronics Engg Dept. \\ S.V. National Inst of Tech \\ Surat, India
}

\begin{abstract}
This paper addresses an intrinsic rule-based license plate localization (LPL) algorithm. It first selects candidate regions, and then filters negative regions with statistical constraints. Key contribution is assigning image inferred weights to the rules leading to adaptability in selecting saliency feature, which then overrules other features and the collective measure, decides the estimation. Saliency of rules is inherent to the frame under consideration hence all inevitable negative effects present in the frame are nullified, incorporating great deal of flexibility and more generalization. Situations considered for simulation, to claim that the algorithm is better generalized are, variations in illumination, skewness, aspect ratio and hence the LP font size, vehicle size, pose, partial occlusion of vehicles and presence of multiple plates. Proposed method allows parallel computation of rules, hence suitable for real time application. The mixed data set has 697 images of almost all varieties. We achieve a Miss Rate $(\mathrm{MR})=\mathbf{4 \%}$ and False Detection Rate $(\mathrm{FDR})=\mathbf{5 . 9 5 \%}$ in average. Also we have implemented skew correction of the above detected LPs necessary for efficient character detection.
\end{abstract}

Keywords- License plate localization; Salient rules; Connected Region Analysis; statistical inconsistency; skew correction.

\section{INTRODUCTION}

License Plate Localization (LPL) is being paid significance day by day, due to the exponential increase of traffic, requiring installation of smart traffic monitoring system. Applications like automatic toll collection, criminal chase, security control of restricted area and traffic law enforcement have been benefited from LPL system. Although experts have undertaken vast and in-depth research over the past many years for developing LPL technique, it is still driven forward due to demand for handling complex conditions arisen due to collective adverse happenings. To highlight, some of them are variations in LP size and associated font styles, time to time alteration in the standardization and vehicles with dissimilar standard LPs sharing the traffic, variations in road curvature, lanes and lighting, non standard vehicle with occlusion and difficulties in camera positioning.

This work aims at developing intelligent LPL algorithm for monitoring Indian traffic. By law all LPs in India are required to be of standard format consisting of 9 to 12 characters as shown in figure 1 . The first two letters identify the state code, followed by two numbers to identify the district. This is often followed by a series code, e.g. 14E, is the fourteen series of private cars and $2 \mathrm{M}$ is the second series of motor bikes.
Recently many states have been adapting a dual letter series for example $\mathrm{CA}, \mathrm{CB}, \mathrm{CC}$ for cars and $\mathrm{MA}, \mathrm{MB}, \mathrm{MC}$ for bikes. Finally a 4 digit number is used to uniquely identify the vehicle. In some states like Delhi tertiary and Gujarat initial ' 0 ' of the district code is omitted.

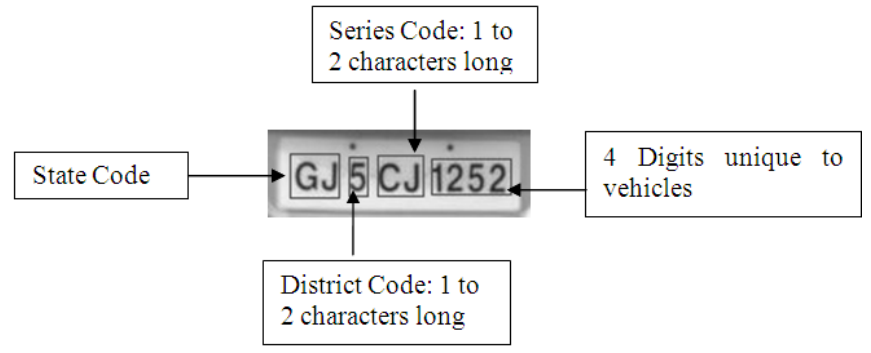

Figure 1. Typical LP pattern in India

\section{STATE OF THE ART}

Prototype LPL systems came into functioning in early eighties. The era began with electronic circuital approach and gradually bowed into computer based processing for real time application. This field has been researched rigorously for more than three decade and many commercial systems are in use in developing countries. However, robust solutions able to face real-world scenarios still need to be proposed. Most of the existing commercial solutions are only applicable to controlled conditions of illumination, viewpoint, or require highresolution and specialized imaging hardware, possibly combined with infrared strobe lights.

Techniques based upon combinations of edge statistics and mathematical morphology [1-4] featured good results. Main disadvantage of this hybrid technique is that edge based methods alone can hardly be applied to complex images, since they are too sensitive to unwanted edges, which may also show a high edge magnitude or variance (e.g., the radiator region in the front view of the vehicle). When combined with morphological steps that eliminate unwanted edges in the processed images, the LP extraction rate becomes relatively high and fast. Though morphological deal along with edge analysis gives good results, it is limited to the fixed distance and angel between camera positions to vehicle as well as it is fail for plates of different size and more than one vehicle in single frame. 
In [5], a block based recognition system was proposed to extract and recognize license plates of motorcycles and vehicles on highways only, in which as a first stage, a blockdifference method was used to detect moving objects and then a screening method, based on the projection of edge magnitudes was used to find two peaks in the projection histograms to find license plates. But main shortcoming of this method is high FDR because of projection of edges.

Former class of algorithm uses knowledge guided train and test approach like support vector machine (SVM) [6] and Adaboost [7]. In SVM, the system uses a window of small size to scan frame and classify the center pixel either for LP or background region by analyzing properties like color and texture. In Adaboost [7] method, first training is planned for classifier using a set of LP and non-LP images and then test frame is fed in to the system, to detect the location of plate in the test image. Knowledge guided training approach is highly depended on training data set and it is not clear how well the detector will generalize in other situations. Haar like features are generally use for vertical and horizontal direction analysis in association with classifiers. Training based methods are also slower compared to edge-based methods.

In $[8,9]$, wavelet feature analysis is applied to identify LP regions, which is helpful to significantly highlight the vertical edges of license plates because of its multi-scale property which enables detection of plates in different scales. Then, LP can easily be extracted by first-order local recursive Otsu segmentation [10] and orthogonal projection histogram analysis.

In Hough transform (HT) based method for license plate extraction, edges in the input image are detected first. Then, HT is applied to detect the LP regions. In [11], a combination of Hough transform and contour algorithm was applied on the edge image. Then the lines that cross the plate frame were determined and a rectangular-shaped object that matched the license plate was extracted. In [12] scan and check algorithm was used followed by radon transform for skew correction.

Representation of color in an invariant manner is of main objectives for color-based object recognition system and therefore based on human perception this feature is very powerful for object recognition. However, the sensitivity of this feature to the parameters such as color of car, illumination condition and the quality of imaging system has been restricted its usage as well as it is computationally complex [13].

Foundation of all the above literatures is vertical edge analysis and hence they are not robust to all natural variations in traffic imaging conditions. Standalone algorithm to handle high range of ambient light, skewness, font size and aspect ratio with no external association is indispensable. Additional features like low computation complexity leading to real time processing is appreciable.

Section-3 of proposed algorithm describes the preprocessing necessary for probable candidate region selection, followed by section-4, illustrating rules and adaptive saliency measure approach. Results obtained under various adverse conditions, to justify the robustness claimed earlier are shown in section-5. Section 6 deals with skew correction part of detected LPs. Section-7 derive the conclusion.

\section{PREPROCESSING}

LPL step is responsible for transformation of data between the real environment and information system. Aim is to extract semantics accurately, for which preprocessing becomes obligatory. We have considered a two-step preprocessing. In first stage for contrast enhancement, algorithm proposed in [14] is applied in which statistical analysis was done for reducing the processing time and if outcome is low, sigmoid transformation is applied for contrast enhancement and then second stage searches for probable candidate region based on vertical edge density and region connectivity. Figure 2 shows the flow chart of proposed algorithm.

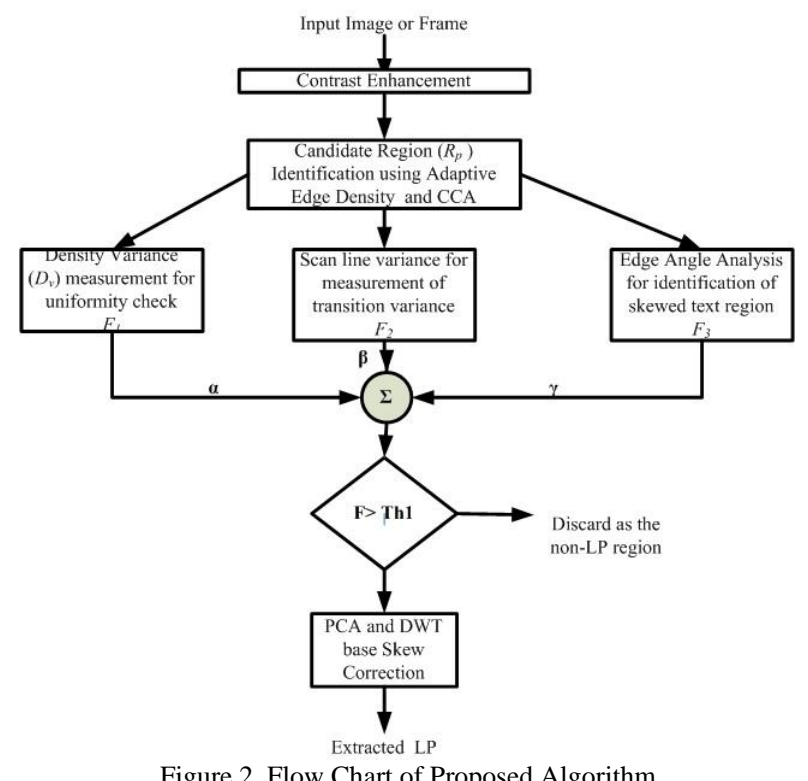

Figure 2. Flow Chart of Proposed Algorithm

\section{A. Candidate Region Identification}

Edges display irregularities in intensity due to presence of salient features. In this application the vertical gradient image is obtained using the x-direction Sobel operator, which is further binarized using Otsu's concept [10] of optimal threshold $k^{*}$, to maximize the between class variance $\sigma_{B}{ }^{2}\left(k^{*}\right)$

$$
\text { Where } \sigma_{B}^{2}\left(k^{*}\right)=\max _{0 \leq k \leq L-1} \sigma_{B}^{2}(k)
$$

The LP also contains the characters of almost equal shape and size and is placed at regularly spaced intervals. In this method a mask of pre-defined size $(w \times h)$ is made to budge over the entire image. The edge density is calculated using (1).

$$
\begin{aligned}
& \text { Edge_den }=\frac{1}{(w \times h)} \sum_{x_{i}}^{x_{i}+w} \sum_{y_{j}} \operatorname{edge}\left(x_{i}, y_{j}\right) \\
& \text { where } x_{i}=x_{i-1}+k_{1} ; y_{j}=y_{j-1}+k_{2} ; \\
& \text { and } \operatorname{edge}\left(x_{i}, y_{j}\right) \in\{0,1\}
\end{aligned}
$$


Where $k_{1}$ and $k_{2}$ decides the mask overlapping along horizontal and vertical direction respectively. If Edge_den is higher than threshold, region covered by mask is considered as probable candidate of LP. The size of mask is decided according to the dynamic range between camera and traffic locality under analysis, which also decides the probable distance of vehicle from the camera and aspect ratio of LP and character size. The height of the mask is computed experimentally from maximum expected plate character height Max_char_ht and minimum expected plate character height Min_char_ht as shown in (2). Purpose of choosing two different parameters is to consider two row plates, which are very common in Indian scenario.

$$
\begin{gathered}
\text { mask }{ }_{-} h t=h=\left\{\frac{\left(\text { Max_char } h t+\text { Min_char }_{-} h t\right)}{2} * \eta\right\} \\
\text { mask_width }=w=\frac{h}{A R_{-} l p}
\end{gathered}
$$

Where, $0.5<\eta<0.9$

As shown in figure 3, white rectangle is the LP region; gray rectangles are masks with different $\eta$ values. Small values of $\eta$ will upshot missing of LP, if vehicle is close to camera. Similarly for large value of $\eta$ probable LP region will come with additional back ground, which may affect the detection accuracy.

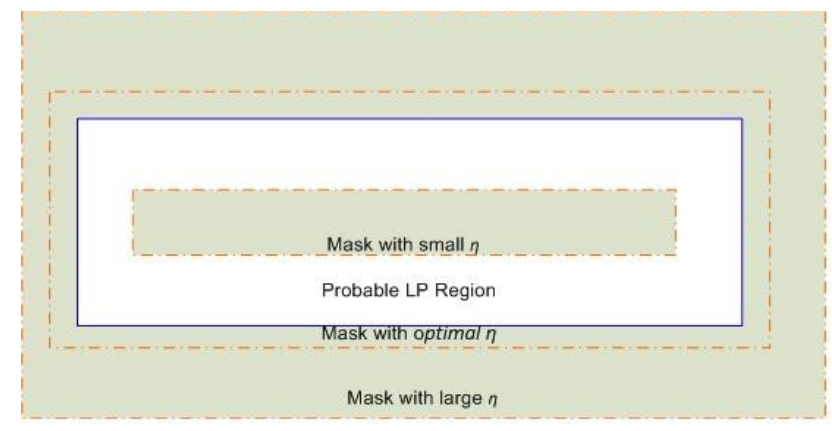

Figure 3: Importance of $\eta$

Figure 4 shows the vertical-gradient image as a thermo graphic map, where "hot" spots denote areas with high vertical edge density which includes all LP regions.
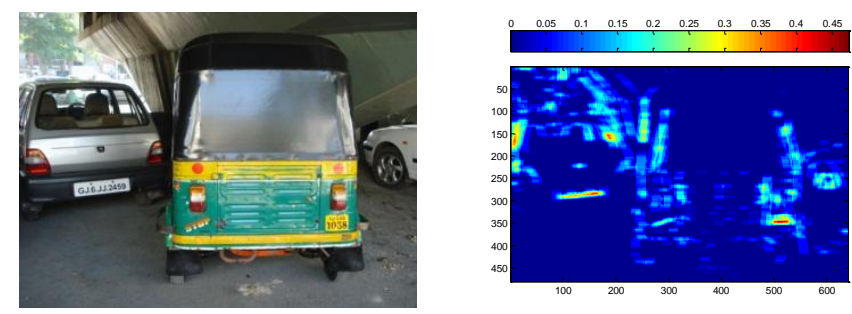

Figure 4. Original image and its thermo graphic image

\section{B. Connected Region Analysis (CRA)}

Connected Region Analysis is performed on probable regions, with 8- connectivity test performed for edge pixels of the regions. If $R_{n}$ and $R_{n+N}$ (where $1<N<8$ ) are two connected regions, CRA will merge them to one region of larger size. Figure 5 shows the possible region connectivity and the outcome after CRA for two blocks.

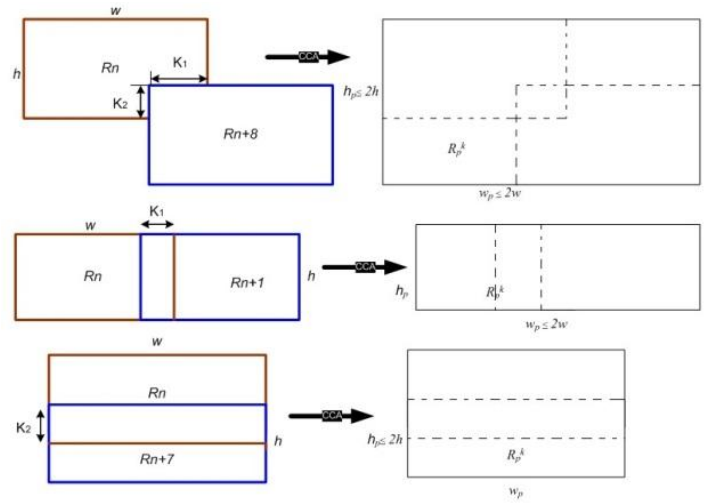

Figure 5. Formation of $R_{p}{ }^{k}$ after CCA

\section{Proposed Rules And SAliency Evaluation}

Next task is to filter out the non-LP regions from the connected probable regions and this is analyzed by setting required statistical properties. We have considered three rules, sufficient to handle almost all complex situations which can be processed in parallel for real time implementation.

\section{A. Density Variance Rule}

License plate consists of not only the copious edge information, but the foreground characters are also usually distributed with relatively even interval. Therefore, for block containing text, the foreground pixels distributed uniformly in the blocks and this feature is useful to discriminate text blocks from graphic blocks. Figure 6 shows the vertical gradient distribution both in LP and non-LP regions.
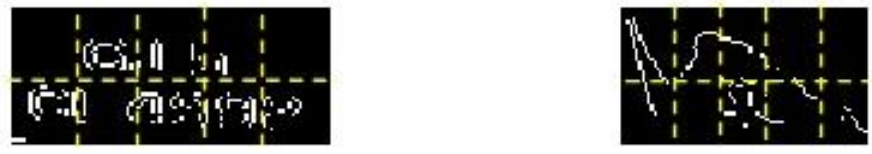

Figure 6. Signature of gradient variation in LP and Non-LP region

For finding out density variance, we segregate block $R_{p}$ in to $n$ equal sized sub blocks $B_{i}$, where $1<i<n$ and $n=10$ for our case. Then density variance and mean value $B_{\text {mean }}$ of block $R_{p}$ is calculated using (3) and (4) respectively.

$$
D_{v}=\frac{\sum_{i=1}^{n}\left|B_{\text {mean }}-B_{i}\right|}{B_{\text {mean }}}
$$

and

$$
B_{\text {mean }}=\frac{1}{n} \sum_{k=1}^{n} B_{k}
$$

From (3), $D_{v}$ remains low for uniform gradient distribution across the block $R_{p}$. 


\section{B. Scan Line Variance Rule}

LPs consists of English alphabets subscribed on contrast background, hence possess a definite edge distribution pattern. The transition between plate background and characters, such as the ones shown in figure 7, are used for inference derivation. To reduce complexity as well as to improve accuracy, top and bottom $20 \%$ of rows are discarded with an assumption that characters on bounding box are centrally located.

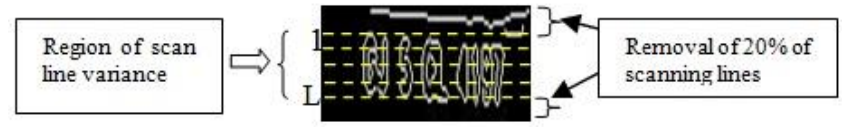

Figure 7. Concept of scan line calculation

Eq. (5) formulates the scan line variance, where $T_{i}$ is the number of transitions in the $i$-th row.

$$
\text { scan_vr }=\sum_{i=1}^{L}\left(T_{i}-T_{a v g}\right) / T_{a v g}
$$

Where $\quad T_{a v g}=\frac{1}{L} \sum_{i=1}^{L} T_{i}$

Main limitation of this parameter is that it is fail to detect skew LP where numbers of transitions may be less due to oblique view leading to fusion of characters.

\section{Edge Orientation Rule}

The distribution of edges is more directional in text blocks, i.e. edge direction histogram corresponding to text block will have maxima at $0^{\circ}$ and $90^{\circ}$. For each edge pixel direction $\psi$ is obtained using (6)

$$
\psi=\arctan \left(g_{v} / g_{h}\right)
$$

Here $g_{v}$ and $g_{h}$ are gradient magnitudes of edge pixels. Gradient angle $\psi$ is quantized $Q(\psi)$ into integer number of bins. Direction bin counters (dir_i) are then incremented if $Q(\psi)$ falls in the bin. For example eight possible directions, $\theta_{1}$ to $\theta_{8}$, with bin span of $22.5^{\circ}$, are as shown in figure 8 .

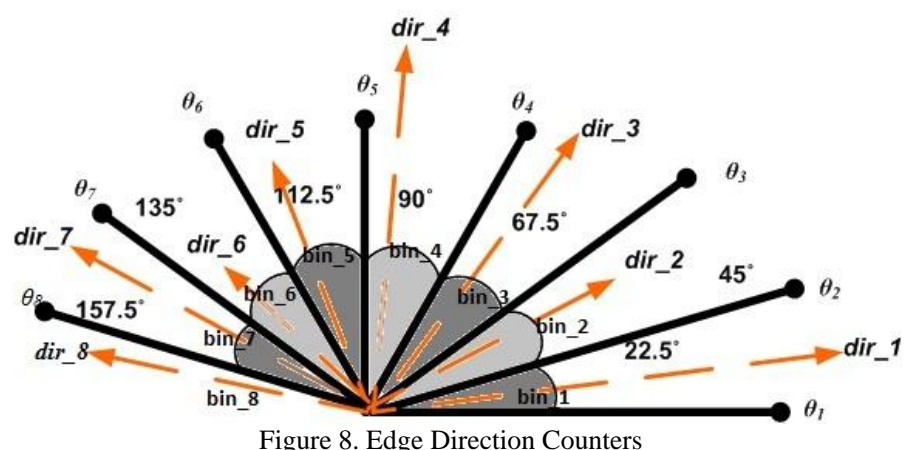

A selection means employed on each edge pixel to increment the corresponding counter is,

$$
\begin{aligned}
& \begin{array}{l}
\theta_{i}=Q\left(\psi / \theta_{S}\right) \text { for } \psi \leq 180^{\circ} \\
\quad=Q\left\{\left(\psi-180^{\circ}\right) / \theta_{S}\right\} \text { for } \psi>180^{\circ}
\end{array} \\
& \text { where } \theta_{S}=180^{\circ} / 8
\end{aligned}
$$

For LP block, assignment of edge pixels for dir_l (horizontal edges) and dir_5 (vertical edges) are maximum compared to other counters as shown in figure 9. Based on this we have used the third rule as given in (8), which supports extraction of tilted LPs.
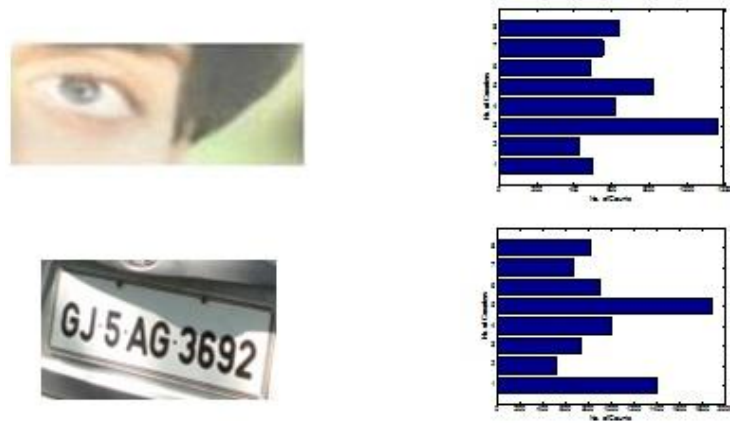

Figure 9. Histogram of counters for Non-text and Text blocks

$$
\begin{aligned}
& \text { edge } e_{-} \text {ori_vr }=\frac{1}{\text { Mean_count }}\left\{\left[\operatorname{dir} r_{-} 1+d i r_{-}\left(\frac{M}{2}+1\right)\right]-\sum_{\substack{n=1 \\
n \neq 1, \frac{M}{2}+1}}^{M} d i r_{-} n\right\} \\
& \text { where Mean_count }=\frac{1}{n} \sum_{n=1}^{M} \operatorname{dir}{ }_{-} n
\end{aligned}
$$

\section{Saliency Evaluation of Rules}

After defining the mathematical formulation of three inconsistency measures, namely (1) density variance $\left(F_{1}\right),(2)$ scan line variance $\left(F_{2}\right)$ and (3) edge orientation variance $\left(F_{3}\right)$, next task is to examine rule saliency and assign related weightage $\alpha, \beta$ and $\gamma$ respectively.

The dynamic range for selection of $\beta$ is computed using (9) using the ratio of $\operatorname{count}(T)$ of scan lines with transitions above a predefined value to the total number of scan lines $(N)$, and aspect ratio of probable region under consideration.

$$
\beta=1 / 3 *\left\{\frac{T}{N}+\frac{h_{p}}{w_{p}}\right\}
$$

Indian LPs consist of 9 to 12 characters therefore block consisting of few text lines can pass through rule $F_{3}$ but it will be blocked by rule $F_{2}$ due to insufficient edge information. Therefore $\gamma$ is determined taking edge orientation into account as shown in (10).

$$
\text { If } \frac{d i r_{-} 1+d_{-} r_{-}\left(\frac{M}{2}+1\right)}{\text { Total_count }}>0.5
$$




$$
\text { then } \gamma=1 / 3 *\left\{\frac{\operatorname{dir}_{-} 1+\operatorname{dir}_{-}\left(\frac{M}{2}+1\right)}{\text { Total_count }}+\frac{h_{p}}{w_{p}}\right\}
$$

else $\gamma=0.2$

' $\gamma$ ' is minimum for erected view and maximum for oblique view. Finally factor $\alpha$ for $F_{1}$ is decided to satisfy the normalization criteria as

$$
\alpha=1-(\beta+\gamma)
$$

Amalgamated rule $F$ then becomes,

$$
F=\alpha^{*} F_{1}+\beta^{*} F_{2}+\gamma^{*} F_{3}
$$

If block under consideration has $F$ more than experimentally set threshold (in our case it is 5.9) then it is selected as LP region, else is discarded.

\section{EXPERIMENTATION RESULTS}

Our database for experimentation consists of 237 low contrast images, consisting of 442 LPs that includes commercial or cargo transport vehicles, motor cycles, auto rickshaw and more than two vehicles in a frame, named as set2. In order to compare the performance unchanged method is also applied on another set consisting of fine images with 707 LPs that is set-1.Two performance measure parameters are:

1. Miss Rate (MR), ratio of number of miss plates (NM) out of total number of detected plates (NDP).

$$
M R(\%)=\frac{N M}{N D P s} * 100
$$

2. False Detection Rate (FDR) which checks accuracy of system by taking ratio of number of false detection (NFD) to NDP.

$$
F D R(\%)=\frac{N F D}{N D P S} * 100
$$

Table 1 gives the performance measure of proposed algorithm on both sets and figure 10 shows the extraction of

\begin{tabular}{|c|c|c|c|c|}
\hline \multirow[b]{2}{*}{$\begin{array}{c}\text { Param- } \\
\text { eters }\end{array}$} & \multicolumn{2}{|c|}{ Analysis on set-1 } & \multicolumn{2}{|c|}{ Analysis on set-2 } \\
\hline & $\begin{array}{l}\text { Proposed } \\
\text { work }\end{array}$ & $\begin{array}{c}\text { Algorithm } \\
\text { of [15] }\end{array}$ & $\begin{array}{l}\text { Proposed } \\
\text { work }\end{array}$ & $\begin{array}{c}\text { Algorithm } \\
\text { of [15] }\end{array}$ \\
\hline$\% \mathrm{MR}$ & 3.68 & 6.5 & 4.43 & 8.5 \\
\hline \%FDR & 5.1 & 8.2 & 6.8 & 10.2 \\
\hline
\end{tabular}
plate for some unfavorable cases. We have compared proposed algorithm with [15], as our inclination is more towards Indian conditions and achieved impressive improvement.

TABLE I. ANALYSIS OF RESULTS USING PROPOSED ALGORITHM
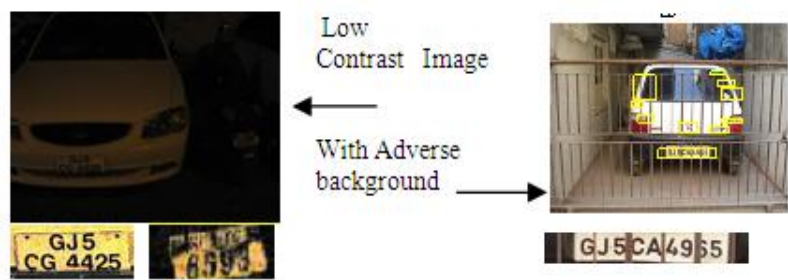

GJ5|CA| $49 \$$ \$
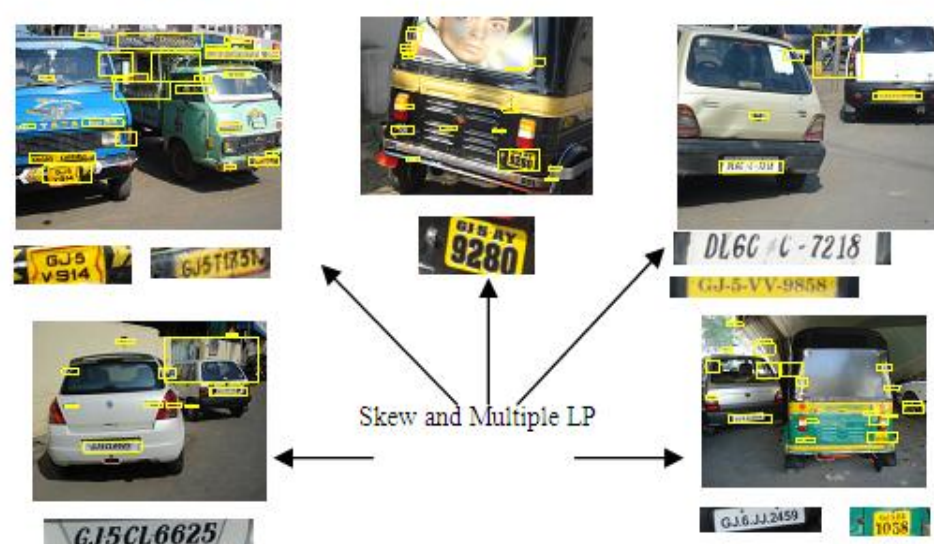

GJSCL6625

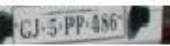

Figure 10. Results with some typical cases

\section{SKew Correction TeChNique}

The skewed license plate affects badly on the accurate character segmentation and recognition. To solve the problem, an efficient approach for skew correction of license plate is proposed based on wavelet transform and principal component analysis. Figure 11 shows the representation of proposed algorithm for skew correction.

By decomposing an extracted LP with 2D-DWT with 'Haar' as a mother wavelet, four frequency sub bands $L_{1}$, $\mathrm{LH}_{1}, \mathrm{HL}_{1}$ and $\mathrm{HH}_{1}$ are obtained. Again by taking second level of decomposition we have achieved bands $\mathrm{LL}_{2}, \mathrm{LH}_{2}, \mathrm{HL}_{2}$ and $\mathrm{HH}_{2}$. Main criterion over here is to find out exact discontinuity in the image, i.e. no edge will be missing. From experimentation, we have short listed 'Daubechies' (DB3 onwards) and 'Haar' wavelets out of other available wavelet families. The final scaling value in the 'Daubechies' transform is not the average of the data set, as it is in the case of the Haar transform, this results that the low pass filter part of the 'Daubechies' transform does not produce as smooth a result as the Haar transform. Finally, we have selected 'Haar' as mother wavelet because of the fact that processing has to be done on binary image and there are only two transitions in 'Haar' wavelet. 'Haar' wavelet will be well suited to binary images and it does not require any complex calculation. Also computational complexity is also very less in 'Haar' compare to 'Daubechies'. 


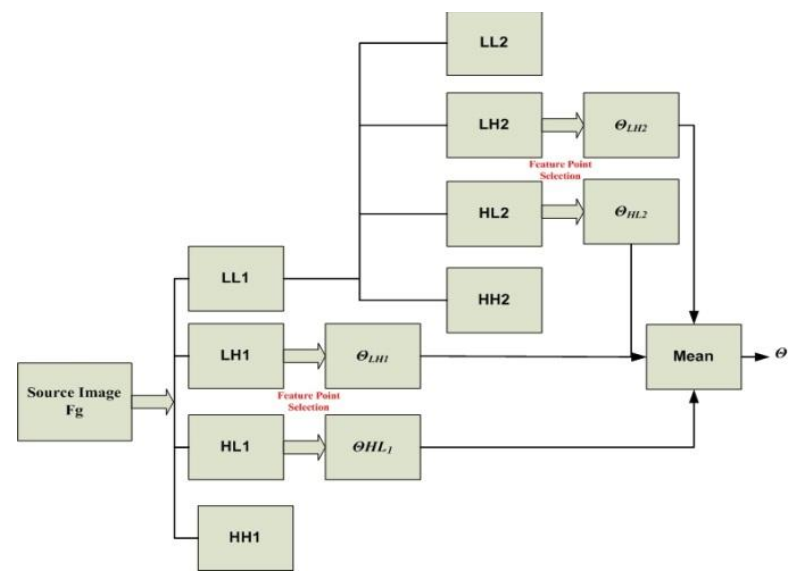

Figure 11. Representation of Skew Correction Algorithm.

Here we are using bands $\mathrm{LH}_{1}, \mathrm{HL}_{1}, \mathrm{LH}_{2}$ and $\mathrm{HL}_{2}$ for feature point selection, as it preserves horizontal and vertical details contained in the image, as well as we get good approximation of the results. Next step is to set a threshold value to binarize the levels obtained after wavelet analysis. White pixels in the image are the extracted feature points as shown in figure 12. Measurement of the two variables $x_{1}$ and $x_{2}$ are considered and measured data under consideration is within the coordinate system $\left(x_{1} \mid x_{2}\right)$, and then the transform it into new coordinate system $\left(z_{1} \mid z_{2}\right)$ using principal component analysis (PCA). The new set of values is examined; the direction in which data are maximal is the direction of principal component $z_{1}$ and the one with smaller variations is $z_{2}$. Therefore component with the smaller variations is neglected.

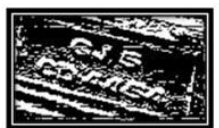

LH1

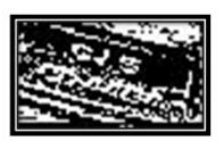

LH2

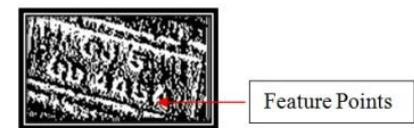

HL1

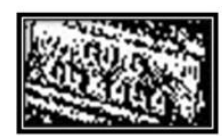

HL2
Figure 12. Feature Points of different Bands

For PCA, initially we have to compute the mathematical expectation for feature points in both $\mathrm{x}$ and $\mathrm{y}$ coordinates using (15) and using it covariance matrix $C_{x}$ will be calculated using (16). Finally eigen values $\lambda_{j}$ and feature vectors $P_{j}$ are computed using (17) and by arranging eigen values in descending order gives the transformation kernel matrix $\mathrm{H}$ using (18).

$$
\begin{gathered}
M_{x 1} \approx \frac{1}{N} \sum_{k=1}^{N} x_{1 k}, M_{x 2} \approx \frac{1}{N} \sum_{k=1}^{N} x_{2 k} \\
C_{x}=\frac{1}{N} \sum_{k=1}^{N}\left(x_{1 k}-M_{x 1}\right)\left(x_{2 k}-M_{x 2}\right)^{T} \\
\left|\lambda_{j} I-C_{x}\right|=0
\end{gathered}
$$

Where $I$ is an identity matrix, $\lambda_{j}=\left(\lambda_{1}, \lambda_{2}, \ldots . \lambda_{j}\right)$ and $\lambda_{1}>\ldots>$ $\lambda_{j}>\ldots>\lambda_{k}$. The corresponding feature vector of $\lambda_{j}$ is $P_{j}$ and $P_{j}=\left[P_{j 1}, P_{j 2} \ldots P_{j k}\right]^{T}$.

$$
H=\left[\begin{array}{l}
P_{1}^{T} \\
\ldots \\
P_{k}^{T}
\end{array}\right]=\left[\begin{array}{ccc}
P_{11} & \ldots & P_{1 k} \\
\ldots & \ldots & \ldots \\
P_{k 1} & \ldots & P_{k k}
\end{array}\right]
$$

The new vector set $\mathrm{Y}$ is obtain by performing PCA as shown in (19)

$$
Y=H(X-M x)
$$

Since mathematical expectation of (19) is zero, its origin of coordinates has moved to the central position which results in to new covariance matrix $C_{x_{-} \text {new }}$, as mention in (20).

$$
C_{x_{-} \text {new }}=\left[\begin{array}{ccc}
\lambda_{1} & \ldots & 0 \\
\ldots & \ldots & \ldots \\
0 & \ldots & \lambda_{k}
\end{array}\right]
$$

The feature vector $P_{l}$, correspondence to most significant eigen value $\lambda_{l}$ (which has maximum value) gives the direction of principal component $z_{l}$ as shown in figure 13. PCA turns the original coordinates into the variance maximum direction and enables the variance of each pixel coordinate projection along projected direction to achieve the maximum.

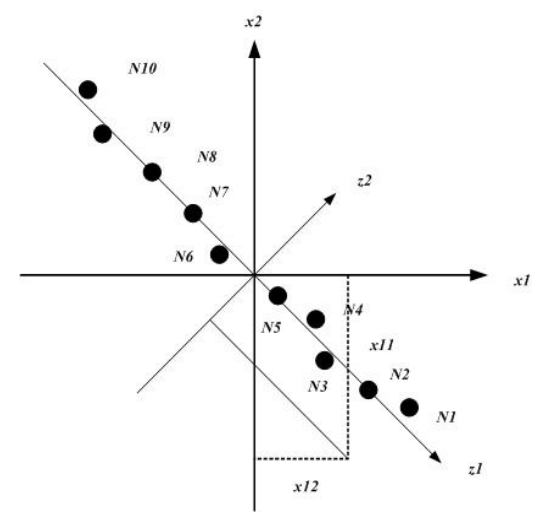

Figure 13. Plot of $\mathrm{N}$ measurements of principal components with two (horizontal and vertical) variables

After 1st level of decomposition (for $\mathrm{LH}_{1}$ ) by computing and arranging eigen values $\lambda_{1}$ and $\lambda_{2}$ in descending order and computing eigen vectors $P_{1}$ and $P_{2}$, rotation angle $\Theta_{L H I}$ will be obtained using (21).

$$
\theta_{L H 1}=\cos ^{-1}\left(P_{1}\right)
$$

Similar procedure has to do for $\mathrm{HL}_{1}, \mathrm{LH}_{2}$ and $\mathrm{HL}_{2}$ and compute $\Theta_{H L 1}, \Theta_{L H 2}$ and $\Theta_{H L 2}$ respectively. Finally rotation by using bilinear interpolation is taken place by taking mean of all four angles. Table 2 shows the computation of rotation angles for image under consideration and figure 14 gives the original skewed and de-skewed LP after skew correction. Figure 15(a) and (b) shows the results of positive and negative skew correction respectively 
TABLE II. COMPUTATION OF ROTATION ANGLE

\begin{tabular}{|c|c|c|c|c|}
\hline Comp. & $\mathbf{L H}_{\mathbf{1}}$ & $\mathbf{H L}_{\mathbf{1}}$ & $\mathbf{L H}_{\mathbf{2}}$ & $\mathbf{H L}_{\mathbf{2}}$ \\
\hline $\boldsymbol{\lambda}_{\boldsymbol{1}}$ & 470.061 & 449.483 & 130.880 & 109.652 \\
\hline$\lambda_{\boldsymbol{2}}$ & 1476.443 & 1411.645 & 391.877 & 362.326 \\
\hline $\boldsymbol{P}_{\boldsymbol{I}}$ & {$[-0.9654$} & {$[-0.9951$} & {$[-0.9687-$} & {$[-0.9929$} \\
& $-0.2607]$ & $0.0986]$ & $0.2481]$ & $0.1192]$ \\
\hline $\boldsymbol{P}_{\boldsymbol{2}}$ & {$[-0.2607$} & {$[0.0986$} & {$[-0.2482$} & {$[0.1192$} \\
& $0.9654]$ & $0.9951]$ & $0.9687]$ & $0.9929]$ \\
\hline $\boldsymbol{\Theta}$ & 15.1092 & 5.6576 & 14.3692 & 6.8471 \\
\hline
\end{tabular}

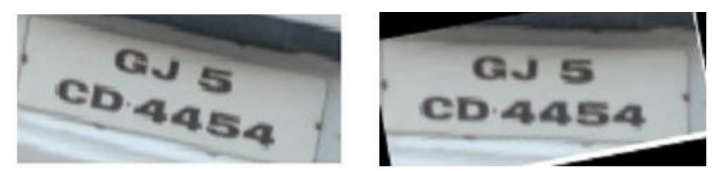

Figure 14 (a) Original Image (b) De skewed Image

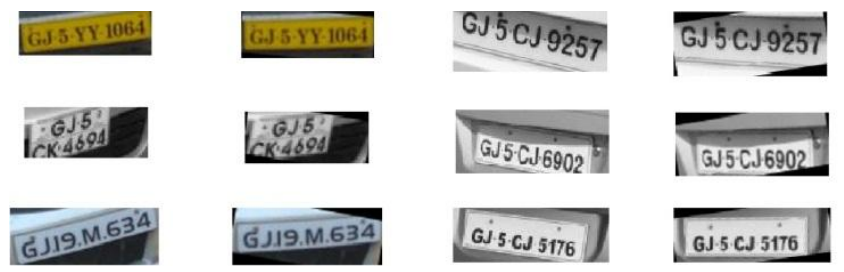

Figure 15 (a) Positive Skew Correction (b) Negative Skew Correction

\section{CONCLUSIONS}

This work presents LPL method based on statistical rules and saliency of rules which gives comparable performance under complex adverse condition. The robustness of proposed method is demonstrated by applying it on a mixed data set with high diversity. Advantage of the proposed algorithm is that the detection box has high precision and it is independent of the detecting license plates with different styles, scales, poses, and partial occlusion. Additional merit is proposed method supports parallel processing and suitable for real time computation. For modifying proposed algorithm for specific country, changes have to be made in Max_char_ht, Min_char_ht and threshold selection for deciding $F$.

In this paper we have also suggested post processing of detected LP regions to nullify the skew error. Skew corrected images then can readily be passed through character detectors for LP number extraction. Image features are view point dependent. Hence normalization of extracted LPs to prescribed size along with orientation would improve the performance. We leave this issue to be considered in future study. Our parallel feature filtering approach reduces the computation time reasonably, however in order to make the system applicable to real time in less restrictive environment feature selection need to be image intrinsic and independent of view point.

\section{REFERENCES}

[1] F. Martin, M. Garcia and J. L. Alba. "New methods for Automatic Reading of VLP's (Vehicle License Plates)," in Proc. IASTED Int. Conf. SPPRA, pp: 126-131, 2002.
[2] C. Wu, L. C. On, C. H. Weng, T. S. Kuan, and K. Ng, "A Macao License Plate Recognition system," in Proc. 4th Int. Conf. Machine Learning Cybernatics, pp: 4506-4510, 2005.

[3] Feng Yang and Fan Yang. "Detecting License Plate Based on Top-hat Transform and Wavelet Transform", ICALIP, pp:998-2003, 2008

[4] Feng Yang and Zheng Ma. "Vehicle License Plate Location Based on Histogramming and Mathematical Morphology", Automatic Identification Advanced Technologies, 2005. pp:89 - 94, 2005

[5] H.-J. Lee, S.-Y. Chen, and S.-Z. Wang, "Extraction and Recognition of License Plates of Motorcycles and Vehicles on Highways," in Proc. ICPR, pp. 356-359, 2004.

[6] K. I. Kim, K. Jung and J. H. Kim, "Color Texture-Based Object Detection: An Application to License Plate Localization", vol. 2388, Springer-Verlag, pp. 293-309.

[7] Xiangdong Zhang, Peiyi Shen, Jianhua Bai, Jing Lei, Yang Hu, Yuli Xiao, Bo Li and Dongpo Qi, "License Plate Detection Using location using AdaBoost", IEEE international conference on Information and Automation, pp:1705 - 1710, 2010.

[8] C.T.Hsieh, Y.-S.Juan and K.-M.Hung, "Multiple license plate detection for complex background", International Conference on Advanced Information Networking and Applications, pp:389-392, 2005.

[9] Chirag Paunwala and Suprava Patnaik, "An Improved License Plate Extraction Technique Based on top-hat transformation and Prolonged Haar Wavelet Analysis", ACM International conference and workshop on Emerging Trends in Technology,pp: 618-622, 2010

[10] N.Otsu, "A Threshold Selection Method from Gray-Level Histograms", IEEE Transactions on System Man and Cybernetics, vol.9(1), pp:62-66, 1979.

[11] T. D. Duan, T. L. H. Du, T. V. Phuoc and N. V. Hoang, "Building an automatic vehicle license-plate recognition system", in Proceddings International Conference on Computer Science, pp. 59-63, 2005.

[12] J. Kong, X. Liu, Y. Lu, and X. Zhou. "A novel license plate localization method based on textural feature analysis," in Proc. IEEE Int. Symp. Signal Process. Inf. Technol., Athens, Greece, pp. 275-279, 2005.

[13] X.Shi, W.Zhao and Y.Shen, "Automatic license plate recognition system based on color image processing", LNCS, vol.3483, pp:1159-1168, 2005.

[14] Chirag Paunwala, Suprava Patnaik, A Novel Multiple License Plate Extraction Technique for Complex Background in Indian Traffic Conditions, Int. Journal of Image Processing, Computer Science Journals, vol.4(2), pp:106-118, 2010.

[15] Prathamesh Kulkarni, Ashish Khatri, Prateek Banga, Kushal Shah, "A Feature Based Approach for Localization of Indian Number Plates", IEEE International Conference on Information Technology, pp: 157162,2009

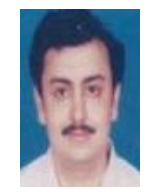

\section{AUTHORS PROFILE}

Prof. Chirag Paunwala received his M.E. in Digital Techniques and Instrumentation from SGSITS, Indore, India in 2006. Presently, he is assistant professor at Electronics and Communication Engineering Department, SCET, Surat, India. His research interest includes Image Processing, Pattern Recognition and Digital Watermarking.

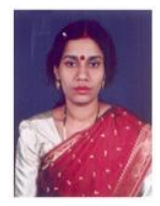

Dr Suprava Patnaik received M.Tech. in Electronic \& Communication Engineering from NIT, Rourkela, in 1992, and Ph.D. in Electrical, Electronics \& Communication Engineering Deprtment from the IIT, Kharagpur in 2004. She is Associate Professor in the Electronics Engineering Department, SVNIT, Surat (Gujarat) India. Her research interests include Signal and Image processing; Image Segmentation, Pattern Recognition, Digital Watermarking, Image Registration \& Video Compression. 